\title{
Erratum: Langevin dynamics and decoherence of heavy quarks at high temperatures [Phys. Rev. C 92, 044911 (2015)]
}

Yukinao Akamatsu $\odot$

(2) (Received 9 October 2019; published 1 May 2020)

DOI: 10.1103/PhysRevC.101.059901

A minor correction regarding Eq. (30) is necessary. Equation (30) is not always correct, for example, with $n^{a}=\delta^{a 8}$ for $N_{c}=3$, one obtains

$$
\left[\vec{f}^{a}(t) \boldsymbol{t}^{a}\right]_{\text {meas }}=\vec{f}(t) \times\left\{\frac{1}{2 \sqrt{3}}, \frac{1}{2 \sqrt{3}},-\frac{1}{\sqrt{3}}\right\} .
$$

Thus, Eq. (30) should read as follows:

$$
\left[\vec{f}^{a}(t) t^{a}\right]_{\text {meas }}=\vec{f}(t) \times\left\{\text { eigenvalues of } n^{a}(t) t^{a}\right\} .
$$

The sentence following Eq. (30) should then read: "There are $N_{c}$ real eigenvalues for a Hermitian matrix $n^{a}(t) \boldsymbol{t}^{a}$," correspondingly. Finally, the sentence a few lines above Eq. (36) should read "For a random pure state, $\left[n^{a}(t) t^{a}\right]_{\text {meas }}$ takes its eigenvalues with equal probabilities." These are all of the necessary modifications.

Equation (32) and below is still valid for the reason given below. Let $e_{\alpha}(t)$ be a column eigenvector of $n^{a}(t) t^{a}$ with an eigenvalue $\lambda_{\alpha}(t)$ for $\alpha=1,2, \ldots, N_{c}$. Then, the (square of) noise strength due to the quantum fluctuation is given by

$$
\begin{aligned}
\vec{f}(t)^{2} \cdot \sum_{\alpha}\left[e_{\alpha}(t)^{\dagger} \rho_{\text {color }}(t ; \zeta) e_{\alpha}(t)\right] \lambda_{\alpha}(t)^{2} & =\vec{f}(t)^{2} \cdot \operatorname{Tr}_{\text {color }}\left\{\boldsymbol{\rho}_{\text {color }}(t ; \zeta)\left(\sum_{\alpha} e_{\alpha}(t) \lambda_{\alpha}(t) e_{\alpha}(t)^{\dagger}\right)^{2}\right\} \\
& =\vec{f}(t)^{2} \cdot \operatorname{Tr}_{\text {color }}\left\{\boldsymbol{\rho}_{\text {color }}(t ; \zeta)\left[n^{a}(t) \boldsymbol{t}^{a}\right]^{2}\right\} \\
& =\text { Eq. (32). }
\end{aligned}
$$

The results and conclusions of the original paper are not affected. 